\title{
COMBINED EXPERIMENTAL POWDER X-RAY DIFFRACTION AND DFT DATA TO OBTAIN THE LOWEST ENERGY MOLECULAR CONFORMATION OF FRIEDELIN
}

\author{
Djalma Menezes de Oliveira, Wagner da Nova Mussel, Lucienir Pains Duarte, Grácia Divina de Fátima Silva, Hélio \\ Anderson Duarte e Elionai Cassiana de Lima Gomes \\ Departamento de Química, Instituto de Ciências Exatas, Universidade Federal de Minas Gerais, Av. Antônio Carlos, 6627, \\ 31270-901 Belo Horizonte - MG, Brasil \\ Luciana Guimarães \\ Departamento de Ciências Naturais, Universidade Federal de São João Del-Rei, Praça Dom Helvécio, 74, 36301-160 \\ São João Del Rei - MG, Brasil \\ Sidney A. Vieira Filho* \\ Departamento de Farmácia, Escola de Farmácia, Universidade Federal de Ouro Preto, Rua Costa Sena, 171, 35400-000 \\ Ouro Preto - MG, Brasil \\ Recebido em 4/1/12; aceito em 9/7/12; publicado na web em 5/10/12

\begin{abstract}
Friedelin molecular conformers were obtained by Density Functional Theory (DFT) and by ab initio structure determination from powder X-ray diffraction. Their conformers with the five rings in chair-chair-chair-boat-boat, and with all rings in chair, are energy degenerated in gas-phase according to DFT results. The powder diffraction data reveals that rings A, B and C of friedelin are in chair, and rings $\mathrm{D}$ and $\mathrm{E}$ in boat-boat, conformation. The high correlation values among powder diffraction data, DFT and reported singlecrystal data indicate that the use of conventional X-ray diffractometer can be applied in routine laboratory analysis in the absence of a single-crystal diffractometer.
\end{abstract}

Keywords: powder X-ray diffraction; conformational study; friedelin.

\section{INTRODUCTION}

Establishing the stereochemistry of organic compounds often represents a challenge for organic chemistry researchers. NMR experiments have been used as an adequate instrument commonly applied for the structural elucidation of compounds such as pentacyclic triterpenes (PCTT). Based on 1D/2D NMR spectral data, the five-ring conformation of PCTT dissolved in deuterated solvent has been established. For pachisandiol, the chair-chair-chair-boat-boat (c-c-c-b-b) conformation was attributed to rings A, B, C, D and E, respectively ${ }^{1}$ and c-c-c-c-c-c ${ }^{2}$ for $16 \alpha$-hydroxy-friedelin, both dissolved in $\mathrm{CDCl}_{3}$. However, it is important to consider the interference of deuterated solvent and the temperature of the analysis in determining the molecular conformation of the compound. This influence is more marked when polar deuterated solvent is used, due to hydrogen bonding and solvation processes.

Another alternative is the use of $a b$ initio Hartree-Fock and density functional theory (DTF) in the conformational studies of PCTT. In this case, the molecule is considered as being in a vacuum, in the absence of any influencing factors.

Single-crystal X-ray diffractometry is also commonly applied to determine the crystal structure of PCTT. A single, pure, stable and representative crystal, with sufficient size to have the overall intrinsic properties of a compound, is necessary for submission to single-crystal $\mathrm{X}$-ray diffractometry for structure elucidation. This necessity becomes more complex for the structural elucidation of natural compounds, such as pentacyclic triterpenes (PCTT), isolated from plants in small amounts, which may not crystallize in this way with a well-defined crystal that represents the overall properties of the natural phase. ${ }^{3}$

Crystal structure determination by powder diffraction data (SDPD) has been widely and successfully applied for organic,

*e-mail: bibo@ef.ufop.br inorganic and organometallic molecules with low molecular mass. ${ }^{4}$ Furthermore, the SDPD procedure of an organic molecule is dependent on some factors related to its crystallinity, purity, size, low conformational energy, polymorphism and some additional chemical characteristics. ${ }^{5}$ It poses a challenge in phytochemistry studies. We present a procedure in which SDPD was applied in the study of friedo-olean-3-one (friedelin) structure (Figure 1a) using Rietveld structure determination combined with theoretical calculations by density functional theory (DFT) ${ }^{6,7}$ and additional NMR spectral data of the compound in $\mathrm{CDCl}_{3}$ solution.
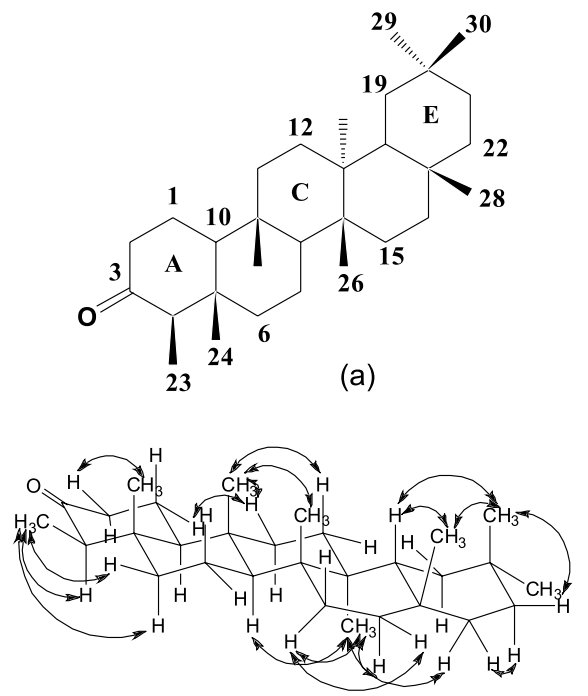

(b)

Figure 1. Chemical structure of friedelin (a) and some hydrogen correlations (b) observed in the NOESY contour maps $\left(\mathrm{CDCl}_{3}, 400 \mathrm{MHz}\right)$ 
This work reports a new approach for crystal structural determination from powder diffraction of friedelin triterpene, associated with NMR spectral data obtained in $\mathrm{CDCl}_{3}$ solution and DFT. It is envisaged that the implementation and optimization of methods commonly found in ordinary laboratories can contribute toward elucidating structural problems of PCTT family compounds, as well as other simple organic molecules, without the need for single-crystal experiments. Previously reported data for a single-crystal of friedelin and NMR studies ${ }^{9}$ were used to validate our results.

\section{EXPERIMENTAL}

\section{Plant material}

A sample of Maytenus acanthophylla (Celastraceae) was collected from the Planalto de Maracás, Maracás, Bahia, Brazil. A voucher specimen was deposited (Collection No. 27182) at the Herbarium of the Botanical Department of the Universidade Federal de Viçosa (UFV), Viçosa, Minas Gerais, Brazil.

\section{Isolation of friedelin}

In the course of the phytochemical study of Maytenus acanthophylla (Celastraceae), a mixture of friedelin and $3 \beta$-friedelinol (2:8) was isolated from their leaves by powder static extraction using hexane as the solvent. These PCTTs were characterized by comparing their ${ }^{13} \mathrm{C}$ NMR data with that previously published ${ }^{10}$. A sample $(1.5 \mathrm{~g})$ was further oxidized ${ }^{11}$ and recrystallized in $\mathrm{CHCl}_{3}-\mathrm{MeOH}$ (8:2), yielding a white solid (1.22 g) of friedelin $\left(\mathrm{C}_{30} \mathrm{H}_{50} \mathrm{O}\right.$; melting point: $259.7-262.1^{\circ} \mathrm{C}$ - Lit. $\left.258.0-260.0^{\circ} \mathrm{C}\right),{ }^{12}$ with $98.5 \%$ purity grade as attested by $\mathrm{CG}$ analysis.

\section{General procedures}

The ${ }^{1} \mathrm{H}$ and ${ }^{13} \mathrm{C}$ NMR spectra were recorded on a Bruker spectrometer operating at $400.13\left({ }^{1} \mathrm{H}\right)$ and $100.62\left({ }^{13} \mathrm{C}\right) \mathrm{MHz}$ at $300 \mathrm{~K}$, with direct $5 \mathrm{~mm}$ detection dual probe ${ }^{1} \mathrm{H} /{ }^{13} \mathrm{C}\left(90^{\circ}\right.$ pulse width - $\mathrm{PW}$ - of $9.8 \mu \mathrm{s}$ and $7.8 \mu \mathrm{s}$ ) for ${ }^{1} \mathrm{H}$ and ${ }^{13} \mathrm{C}$, respectively. The sample (10 mg) was dissolved in $\mathrm{CDCl}_{3}(0.5 \mathrm{~mL})$ and transferred to a $5.0 \mathrm{~mm}$ o.d. NMR tube. TMS was used as the internal standard $\left(\delta_{\mathrm{H}}=\delta_{\mathrm{C}}=0\right)$. NMR data manipulations were carried out on a Bruker Avance DRX-400 with SGI workstation software. Melting points were obtained on a Mettler FP 80 HT apparatus.

\section{DFT calculations}

Theoretical studies for cccc and cccbb friedelin conformers were carried out using the Gaussian 03 program package. ${ }^{13}$ The geometry optimizations were performed using density functional theory (DFT) with the B3LYP functional ${ }^{7}$ employing the standard Pople's split valence $6-31 \mathrm{G}(\mathrm{d}, \mathrm{p})$ basis set. ${ }^{14,15}$ Geometries were characterized as true minima in the potential energy surface (PES) with all harmonic frequencies being real. The energy between the two conformers $\left(\Delta G^{\text {total }}\right)$ was calculated as the sum of two parts: electronic plus nuclear repulsion energy $\left(\Delta E^{\text {ele }}\right)$ and thermal correction to the Gibbs free energy $\left(\Delta G^{\text {therm }}\right)$. The thermal contribution in gas phase was evaluated using the canonical formalism at $298 \mathrm{~K} .{ }^{16}$ The calculated harmonic vibrational frequencies were used to estimate the corrections of zero point energy (ZPE) due to the thermal population of the vibrational levels. The initial geometry optimization of the friedelin molecule started with two conformational forms (cccbb and ccccc) expected for the D:A-friedooleananes ${ }^{17}$ and were carried out with the experimental geometrical parameters for $3 \alpha$-friedelinol ${ }^{18}$ and methyl-3-oxofriedelan-20 $\alpha$-oate, ${ }^{19}$ respectively.
Friedelin sample was size-reduced to a very fine powder and deposited as a film suspension in a Zero Field Sample Holder (ZFSH) composed of polished $\mathrm{SiC}$ in a $3^{\circ}$ angle mount to reduce background contributions to the $\mathrm{X}$-ray diffraction experiment. The material was initially suspended in isopropyl alcohol and homogenously spread over the sample holder under spinning to prevent preferred orientation and to minimize rugosity effects over the exposed surface, and was subsequently left to dry at room temperature.

The powder X-ray diffraction data were collected on a Siemens D5000 diffractometer under 40KV, 30mA, using $\mathrm{Cu} \mathrm{K} \alpha(\lambda=1.54056$ $\AA$ ) coupled with a graphite monochromator, scanned over an angular range of $4-40^{\circ}(2 \theta)$ with a step size of $0.01^{\circ}(2 \theta)$ and a time constant of $15 \mathrm{~s} \mathrm{step}^{-1}$. The sample holder was submitted to a spinning speed of 60 cycles per minute to reduce any preferred orientation and to minimize rugosity effects. The final matrix was averaged over 3 independent scans.

The crystal structure was determined by $a b$ initio calculations. The powder indexing tool used for peak identification, indexing and automatic space group determination was DICVOL91. ${ }^{20}$ The peaks were searched and fitted with a Pseudo-Voigt peak profile, performing both modified Pawley and Rietveld refinement to optimize powder diffraction parameters and crystal structure so that the best possible agreement between simulated and experimental powder patterns was achieved. It crystallized in a P212121 space group with $a=6.371$ $\AA, b=13.943 \AA, c=28.456 \AA, \alpha=\beta=\gamma=90^{\circ}, Z=4$, Rwp $=0.1153 \%$, $\mathrm{Rp}=0.0638 \%$.

Initially the pattern was processed for background contribution; peaks were searched and manually inspected when necessary to prevent background fluctuations from being considered reflections. The molecule was sketched using ChemSketch ${ }^{\circledR}$ (ACD-LabsV.12.0 for Microsoft Windows $\left.{ }^{\circledR}, 2009\right),{ }^{21}$ the hydrogen atoms were introduced based on the NMR spectra and the molecule allowed to have its energy minimized by the Monte Carlo method before using it as a model. The crystal structure solution was first obtained without any preferred orientation correction and close-contact penalty. The results were compared with the fit using Marck-Dollese preferred orientation and close-contact penalty algorithm. The result that combined the lowest final energy obtained by Monte-Carlo optimization was used as the solution. Details of refinements and experimental data of $\mathrm{X}$-ray diffraction are summarized in Table 1.

Table 1. Experimental details of X-ray diffraction

\begin{tabular}{ll}
\hline \multicolumn{1}{c}{ Crystal data } & \\
\hline Chemical formula & $\mathrm{C}_{30} \mathrm{H}_{50} \mathrm{O}$ \\
$M r$ & 426.70 \\
Cell setting, space group & Orthorhombic, \\
Temperature (K) & $298(1)$ \\
$\mathrm{a}, \mathrm{b}, \mathrm{c}(\AA)$ & $6.371,13.943,28.456$ \\
$\alpha=\beta=\gamma$ & 90 \\
Final V $\left(\AA^{3}\right)$ & 2527.77 \\
$\mathrm{Z}$ & 4 \\
$D_{x}\left(\mathrm{Mg} \mathrm{m}^{-3}\right)$ & 1.121 \\
Radiation type, wavelength & $\mathrm{Cu} \mathrm{K \alpha 1}, 1.54056$ \\
Specimen form, color & Powder white \\
Data collection & \\
Diffractometer & Siemems D5000 \\
Data collection method & Disc geometry, transmission mode, step scan \\
20 range min-max, increment & $4-40,0.01,16.7$ \\
$\left(^{\circ}\right)$, total time (h) & \\
\hline
\end{tabular}




\section{RESULTS AND DISCUSSION}

\section{NMR spectral data}

The chemical shifts of ${ }^{1} \mathrm{H}$ and ${ }^{13} \mathrm{C}$ and the correlation data (Table 2) obtained from COSY and NOESY contour maps indicated conformation cccbb (Figure 1b) for friedelin in $\mathrm{CDCl}_{3}$. The COSY contour map revealed the absence of correlations between vicinal hydrogen at $\delta_{\mathrm{H}} 1.28, \mathrm{~m}(\mathrm{H} \alpha-15)$ and at $\delta_{\mathrm{H}} 1.58, \mathrm{~m}(\mathrm{H} \beta-16$, ring $\mathrm{D})$ as well as between the signal at $\mathrm{H} \beta-18\left(\delta_{\mathrm{H}} 1.57, \mathrm{dd}, J=5.0,12.0 \mathrm{~Hz}\right)$ and $\mathrm{H} \beta-19\left(\delta_{\mathrm{H}} 1.38, \mathrm{~m}\right.$, ring E). Conversely, correlations of $\mathrm{H} \beta-18$ and $\mathrm{H} \alpha-19\left(\delta_{\mathrm{H}} 1.21, \mathrm{~m}\right.$, ring $\left.\mathrm{E}\right)$ were observed. The correlations observed in the NOESY contour map of $\beta$-equatorial methyl-23, $\delta_{\mathrm{H}} 0.88$ (d, $J=6.8 \mathrm{~Hz})$ with the signal of H $\beta-6(1.76, \mathrm{dt}, J=2.5,5.0 \mathrm{~Hz})$, as well as between $\mathrm{H} \alpha-1\left(\delta_{\mathrm{H}} 1.97\right.$, tdd, $\left.2.5,7.0,13.0 \mathrm{~Hz}\right)$ and $\mathrm{H} \beta-11$ $\left(\delta_{\mathrm{H}} 1.48, \mathrm{~m}\right)$ (Table 2$)$ provided confirmation of the chair-chair-chair conformation of rings A, B and C (Figure $1 \mathrm{~b}$ ). The intercrossed NOE correlations between the signal of $\mathrm{H} \alpha-15\left(\delta_{\mathrm{H}} 1.28, \mathrm{~m}\right)$ and $\mathrm{H} \beta-16\left(\delta_{\mathrm{H}}\right.$ $1.58, \mathrm{~m})$ and the signals of $\mathrm{H} \beta-15\left(\delta_{\mathrm{H}} 1.50, \mathrm{~m}\right)$ and $\mathrm{H} \alpha-16\left(\delta_{\mathrm{H}} 1.35\right.$, $\mathrm{m}$ ) indicated that $\mathrm{H} \alpha-16$ is at the bottom of ring D. The conformation of ring E was confirmed based on the correlations of methylene hydrogen $\mathrm{H}-19 \delta_{\mathrm{H}} 1.21,(\mathrm{~m}, \mathrm{H} \alpha-19)$ and $\delta_{\mathrm{H}} 1.38$, (m, H $\left.\beta-19\right)$ with the signal of methyl hydrogen $\mathrm{H} \alpha-29$ at $\delta_{\mathrm{H}} 0.95$, and the signals of $\mathrm{H} \beta-18\left(\delta_{\mathrm{H}} 1.57, \mathrm{dd}, J=5.0,12.0 \mathrm{~Hz}\right)$ and $\mathrm{H} \beta-21\left(\delta_{\mathrm{H}} 1.30, \mathrm{~m}\right)$ with $\mathrm{H} \beta-30$ at $\delta_{\mathrm{H}}$ 1.01. Based on the NMR data (Table 2), it was established that friedelin in $\mathrm{CDCl}_{3}$ solution acquires preferential conformation $c c c b$ (Figure 1b).

It is possible to apply these procedures to similar PCTTs in an

Table 2. 1D/2D NMR spectral data of friedelin

\begin{tabular}{|c|c|c|c|c|}
\hline \multicolumn{3}{|c|}{${ }^{1} \mathrm{H}$ and ${ }^{13} \mathrm{C}$ NMR, DEPT-135 and HSQC data } & \multirow{2}{*}{ COSY } & \multirow{2}{*}{ NOESY } \\
\hline C type & $\delta_{\mathrm{C}}$ & $\delta_{\mathrm{H}}[\mathrm{m}, J(\mathrm{~Hz})]$ & & \\
\hline $\mathrm{C}-5$ & 42.16 & & & \\
\hline C-9 & 37.47 & & & \\
\hline $\mathrm{C}-13$ & 39.72 & & & \\
\hline $\mathrm{C}-14$ & 38.32 & & & \\
\hline $\mathrm{C}-17$ & 30.01 & & & \\
\hline $\mathrm{C}-20$ & 28.18 & & & \\
\hline $\mathrm{O}=\mathrm{C}-3$ & 213.22 & & & \\
\hline $\mathrm{HC}-4$ & 58.25 & $\mathrm{H \alpha}-4: 2.25(\mathrm{q}, 6.8)$ & $\mathrm{Me} \beta-23$ & $\mathrm{H} \alpha-10, \mathrm{Me} \beta-23$ \\
\hline $\mathrm{HC}-8$ & 53.13 & $\mathrm{H} \alpha-8: 1.40(\mathrm{~m})$ & $\mathrm{H} \beta-7$ & H $\alpha-10$, Мe $\alpha-27$ \\
\hline $\mathrm{HC}-10$ & 59.51 & $\mathrm{H} \alpha-10: 1.53(\mathrm{dd}, J=2.5,12.5)$ & $\mathrm{H} \alpha-1, \mathrm{H} \beta-1$ & $\mathrm{H} \alpha-1, \mathrm{H} \alpha-4, \mathrm{H} \alpha-8$ \\
\hline HC-18 & 42.82 & $\mathrm{H} \beta-18: 1.57(\mathrm{~d}, J=5.0)$ & $H \alpha-19$ & Н $\beta-21$, Ме $\beta-28$, Ме $\beta-30$ \\
\hline $\mathrm{H}_{2} \mathrm{C}-1$ & 22.30 & $\begin{array}{l}\mathrm{H} \alpha-1: 1.97(\mathrm{tdd}, 2.5,7.0,13.0) \\
\mathrm{H} \beta-1: 1.68(\mathrm{dd}, 5.0,13.0)\end{array}$ & $\begin{array}{c}\mathrm{H} \beta-1, \mathrm{H} \alpha-2, \mathrm{H} \beta-2, \mathrm{H} \alpha-10 \\
\mathrm{H} \alpha-1, \mathrm{H} \alpha-2, \mathrm{H} \alpha-10\end{array}$ & $\begin{array}{c}\mathrm{H} \beta-1, \mathrm{H} \alpha-2, \mathrm{H} \beta-2, \mathrm{H} \alpha-10, \mathrm{H} \beta-11 \\
\mathrm{H} \alpha-1\end{array}$ \\
\hline $\mathrm{H}_{2} \mathrm{C}-2$ & 41.54 & $\begin{array}{l}\mathrm{H \alpha}-2: 2.31(\mathrm{dd}, 7.0,13.0) \\
\mathrm{H} \beta-2: 2.39(\mathrm{dd}, 2.5,5.0)\end{array}$ & $\begin{array}{c}\mathrm{H} \alpha-1, \mathrm{H} \beta-1, \mathrm{H} \beta-2 \\
\mathrm{H} \beta-1, \mathrm{H} \alpha-2\end{array}$ & $\begin{array}{l}H \alpha-1, H \beta-2 \\
H \alpha-1, H \alpha-2\end{array}$ \\
\hline $\mathrm{H}_{2} \mathrm{C}-6$ & 41.32 & $\begin{array}{c}\mathrm{H} \alpha-6: 1.28 \\
\mathrm{H} \beta-6: 1.76(\mathrm{dt}, J=2.5,5.0)\end{array}$ & $\begin{array}{l}\mathrm{H} \beta-6, \mathrm{H} \beta-7 \\
\mathrm{H} \alpha-6\end{array}$ & $\begin{array}{c}\text { Н } \alpha-4, \mathrm{H} \beta-6, \mathrm{Me} \beta-23 \\
\mathrm{H} \alpha-6, \mathrm{H} \alpha-7, \mathrm{H} \beta-7, \mathrm{Me} \beta-23, \mathrm{Me} \beta-24\end{array}$ \\
\hline $\mathrm{H}_{2} \mathrm{C}-7$ & 18.25 & $\begin{array}{l}\mathrm{H} \alpha-7: 1.40(\mathrm{~m}) \\
\mathrm{H} \beta-7: 1.50(\mathrm{~m})\end{array}$ & $\begin{array}{c}\mathrm{H} \beta-7 \\
\mathrm{H} \alpha-7, \mathrm{H} \alpha-8\end{array}$ & $\begin{array}{c}\text { H } \beta-6, \mathrm{H} \beta-7 \\
\mathrm{H} \beta-6, \mathrm{H} \alpha-7, \mathrm{Me} \beta-24\end{array}$ \\
\hline $\mathrm{H}_{2} \mathrm{C}-11$ & $35.65^{\mathrm{a}}$ & $\begin{array}{l}\mathrm{H} \alpha-11: 1.26(\mathrm{~m}) \\
\mathrm{H} \beta-11: 1.48(\mathrm{~m})\end{array}$ & $\begin{array}{c}\mathrm{H} \beta-11, \mathrm{H} \beta-12 \\
\mathrm{H} \alpha-11\end{array}$ & $\begin{array}{c}\mathrm{H} \alpha-8, \mathrm{H} \beta-11, \mathrm{Me} \alpha-27 \\
\mathrm{H} \alpha-1, \mathrm{H} \beta-11, \mathrm{H} \beta-12, \mathrm{Me} \beta-25\end{array}$ \\
\hline $\mathrm{H}_{2} \mathrm{C}-12$ & 30.52 & $\begin{array}{l}\mathrm{H} \alpha-12: 1.19(\mathrm{~m}) \\
\mathrm{H} \beta-12: 1.35(\mathrm{~m})\end{array}$ & $\begin{array}{c}\mathrm{H} \beta-12 \\
\mathrm{H} \alpha-12, \mathrm{H} \alpha-11\end{array}$ & $\begin{array}{c}\text { Me } \alpha-27 \\
\mathrm{H} \beta-11, \mathrm{Me} \beta-25\end{array}$ \\
\hline $\mathrm{H}_{2} \mathrm{C}-15$ & $32.44^{\mathrm{a}}$ & $\begin{array}{l}\mathrm{H} \alpha-15: 1.28(\mathrm{~m}) \\
\mathrm{H} \beta-15: 1.50(\mathrm{~m})\end{array}$ & $\begin{array}{l}\mathrm{H} \beta-15 \\
\mathrm{H} \alpha-15\end{array}$ & $\begin{array}{c}\mathrm{H} \beta-15, \mathrm{H} \beta-16, \mathrm{H} \alpha-22, \mathrm{Me} \alpha-27 \\
\mathrm{H} \alpha-15, \mathrm{H} \alpha-16, \mathrm{Me} \beta-28\end{array}$ \\
\hline $\mathrm{H}_{2} \mathrm{C}-16$ & 36.03 & $\begin{array}{l}\mathrm{H} \alpha-16: 1.36(\mathrm{~m}) \\
\mathrm{H} \beta-16: 1.58(\mathrm{~m})\end{array}$ & $\begin{array}{l}\mathrm{H} \beta-16 \\
\mathrm{H} \alpha-16\end{array}$ & $\begin{array}{l}\mathrm{H} \beta-15 \\
\mathrm{H} \alpha-15\end{array}$ \\
\hline $\mathrm{H}_{2} \mathrm{C}-19$ & $35.36^{\mathrm{a}}$ & $\begin{array}{l}\mathrm{H} \alpha-19: 1.21(\mathrm{~m}) \\
\mathrm{H} \beta-19: 1.38(\mathrm{~m})\end{array}$ & $\begin{array}{c}\mathrm{H} \beta-19, \mathrm{H} \beta-18, \mathrm{Me} \beta-30 \\
\mathrm{H} \alpha-19\end{array}$ & $\begin{array}{l}\text { Н } \beta-19, \text { Me } \alpha-27, \text { Me } \alpha-29 \\
\text { H } \alpha-19, \text { Me } \alpha-29\end{array}$ \\
\hline $\mathrm{H}_{2} \mathrm{C}-21$ & $32.78^{\mathrm{a}}$ & $\begin{array}{l}\mathrm{H} \alpha-21: 1.53(\mathrm{~m}) \\
\mathrm{H} \beta-21: 1.30(\mathrm{~m})\end{array}$ & $\begin{array}{c}\mathrm{H} \beta-21, \mathrm{H} \beta-22 \\
\mathrm{H} \alpha-21\end{array}$ & $\begin{array}{c}\mathrm{H} \beta-21, \mathrm{H} \alpha-22, \mathrm{Me} \alpha-27 \\
\mathrm{H} \alpha-21, \mathrm{H} \beta-18, \mathrm{H} \alpha-22, \mathrm{Me} \beta-28, \mathrm{Me} \beta-30\end{array}$ \\
\hline $\mathrm{H}_{2} \mathrm{C}-22$ & 39.27 & $\begin{array}{l}\mathrm{H} \alpha-22: 0.94(\mathrm{~m}) \\
\mathrm{H} \beta-22: 1.51(\mathrm{~m})\end{array}$ & $\begin{array}{c}\mathrm{H} \beta-22 \\
\mathrm{H} \alpha-22, \mathrm{H \alpha}-21\end{array}$ & $\begin{array}{c}\mathrm{H} \beta-22, \mathrm{H} \alpha-21, \mathrm{H} \beta-21, \mathrm{Me} \alpha-27 \\
\mathrm{H} \alpha-22, \mathrm{Me} \beta-28\end{array}$ \\
\hline $\mathrm{H}_{3} \mathrm{C}-23$ & 6.83 & $\mathrm{Me} \beta-23: 0.88(\mathrm{~d}, J=6.8)$ & $\mathrm{H} \alpha-4$ & $\mathrm{H} \beta-1, \mathrm{H} \alpha-4, \mathrm{H} \alpha-6, \mathrm{H} \beta-6, \mathrm{Me} \beta-24$ \\
\hline $\mathrm{H}_{3} \mathrm{C}-24$ & 14.67 & $\mathrm{Me} \beta-24: 0.73$ (s) & & $\mathrm{H} \beta-7, \mathrm{Me} \beta-23, \mathrm{Me} \beta-25$ \\
\hline $\mathrm{H}_{3} \mathrm{C}-25$ & 17.96 & $\mathrm{Me} \beta-25: 0.87(\mathrm{~s})$ & & $\mathrm{H} \beta-11, \mathrm{H} \beta-12, \mathrm{Me} \beta-24, \mathrm{Me} \beta-26$ \\
\hline $\mathrm{H}_{3} \mathrm{C}-26$ & 20.28 & Мeß-26: 1.00 (s) & & $\mathrm{H} \beta-7, \mathrm{H} \beta-25, \mathrm{Me} \beta-28$ \\
\hline $\mathrm{H}_{3} \mathrm{C}-27$ & 18.67 & Me $\alpha-27: 1.05$ (s) & & $\begin{array}{c}\mathrm{H} \alpha-8, \mathrm{H} \alpha-12, \mathrm{H} \alpha-15, \mathrm{H} \alpha-16, \mathrm{H} \alpha-19, \mathrm{H} \alpha-22 \\
\mathrm{Me} \beta-29\end{array}$ \\
\hline $\mathrm{H}_{3} \mathrm{C}-28$ & 32.10 & Мeß-28: 1.18 (s) & & $\mathrm{H} \beta-15, \mathrm{H} \beta-18, \mathrm{H} \beta-21, \mathrm{H} \beta-22, \mathrm{Me} \beta-26, \mathrm{Me} \beta-30$ \\
\hline $\mathrm{H}_{3} \mathrm{C}-29$ & 35.03 & Me $\alpha-29: 0.95$ (s) & & Н $\alpha-19, \mathrm{H} \beta-19$, Мe $\alpha-27$ \\
\hline $\mathrm{H}_{3} \mathrm{C}-30$ & 31.79 & $\mathrm{Me} \beta-30: 1.01(\mathrm{~s})$ & & $\mathrm{H} \beta-18, \mathrm{H} \beta-21, \mathrm{Me} \beta-28$ \\
\hline
\end{tabular}

a interchangeable signals. ${ }^{* 1} \mathrm{H}\left(\mathrm{CDCl}_{3}, 400 \mathrm{MHz}\right)$ and ${ }^{13} \mathrm{C}\left(\mathrm{CDCl}_{3}, 100 \mathrm{MHz}\right)$, TMS as internal standard. 
attempt to obtain the low energy conformation in solid state as shown in our work.

The optimized structures of cccbb and ccccc conformers for the friedelin molecule are depicted in Figure 2. The $\Delta G^{\text {total }}$ difference between cccbb and ccccc conformers is only $1.4 \mathrm{KJ} \mathrm{mol}^{-1}$, at B3LYP/6$31 \mathrm{G}(\mathrm{d}, \mathrm{p})$ level of theory. An ORTEP $3^{22}$ view of friedelin is shown in Figure 3 . This means that these species are energetically degenerated in gas-phase, in agreement with force field calculations presented by

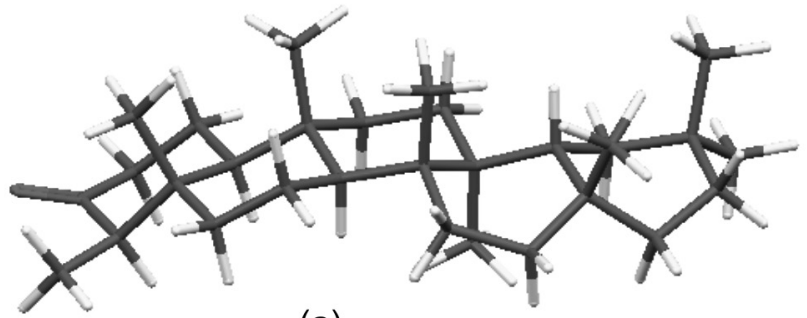

(a)

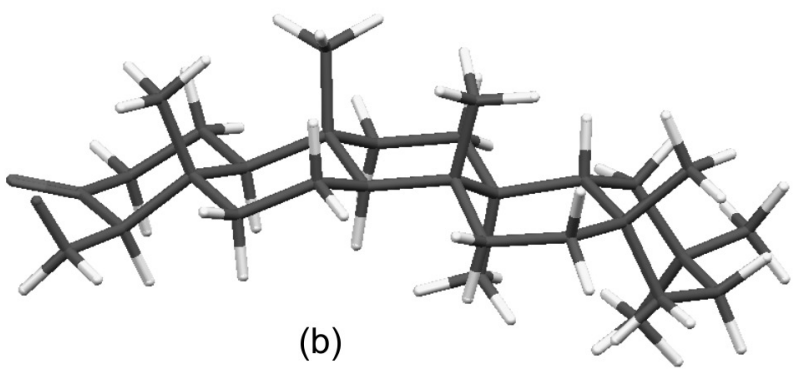

Figure 2. DFT/B3LYP optimized structures of D:A-3-friedoolean-3-one (friedelin): (a) low energy chair-chair-chair-boat-boat (cccbb) conformer and (b) high energy chair-chair-chair-chair-chair (ccccc) conformer

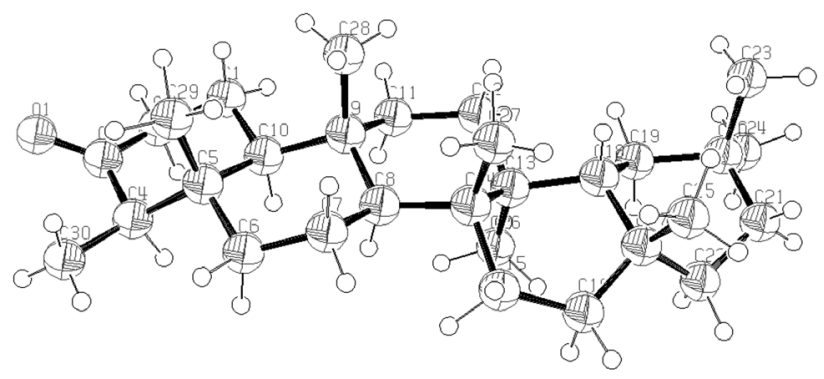

Figure 3. An ORTEP3 view of friedelin. Displacement ellipsoids are draw at $50 \%$ probability level and $H$ atoms shown as small spheres of arbitrary radii

Mo et al. ${ }^{8}$ According to these calculations, the intermolecular forces related to crystal packing, solvation and solubility also determine the preferred conformer in crystalline state.

The calculated and observed values for endocyclic torsion angles for friedelin rings are given in Table 3. A significant agreement was attained where the largest r.m.s deviation between experimental and calculated torsion angles was $2.8^{\circ}$ while an overall deviation of around $1.8^{\circ}$ was verified. The correlation between experimental and calculated values was significantly higher $\left(r^{2}=0.9990\right)$, indicating that the crystallographic solution details of the molecular conformation of friedelin were reproduced with excellent agreement by the DFT calculations.

\section{Powder X-ray diffraction data of friedelin}

The final atomic parameters (atoms coordinate) are listed in Table 4. The final structure solution (Figure 4) obtained by the Rietveld refinement was rationalized by the single-crystal diffraction data previously reported. ${ }^{8}$

Table 3. Comparison of torsion angles $\left(^{\circ}\right)$ as observed (Obs) in powder diffraction experiment with calculated (Calc) by DFT for friedelin structure. The mono data were extracted from Table 1 in ref 23. The observed differences are shown for each set of carbon connection

\begin{tabular}{|c|c|c|c|c|c|c|c|c|c|c|c|}
\hline Ring A & Calc & SDPD & Mono $^{8}$ & $\varepsilon_{1}{ }^{a}$ & $\varepsilon_{2}{ }^{a}$ & Ring D & Calc & SDPD & Mono $^{8}$ & $\varepsilon_{1}{ }^{a}$ & $\varepsilon_{2}{ }^{a}$ \\
\hline $\mathrm{C} 1-\mathrm{C} 2-\mathrm{C} 3-\mathrm{C} 4$ & $-50,8$ & $-53,7$ & $-53.6(7)^{*}$ & 2,9 & $2.8(7)$ & C18-C13-C14-C15 & $-64,8$ & $-67,8$ & $-67.9(6)$ & 3,0 & $3.1(6)$ \\
\hline $\mathrm{C} 2-\mathrm{C} 3-\mathrm{C} 4-\mathrm{C} 5$ & 57,1 & 58,5 & $58.5(7)$ & 1,4 & $1.4(7)$ & $\mathrm{C} 13-\mathrm{C} 14-\mathrm{C} 15-\mathrm{C} 16$ & 27,3 & 30,5 & $30.6(7)$ & 3,2 & $3.3(7)$ \\
\hline $\mathrm{C} 3-\mathrm{C} 4-\mathrm{C} 5-\mathrm{C} 10$ & $-59,8$ & $-58,5$ & $58.5(6)$ & 1,3 & $1.3(6)$ & $\mathrm{C} 14-\mathrm{C} 15-\mathrm{C} 16-\mathrm{C} 17$ & 24,5 & 23,0 & $23.0(8)$ & 1,5 & $1.5(8)$ \\
\hline $\mathrm{C} 4-\mathrm{C} 5-\mathrm{C} 10-\mathrm{C} 1$ & 60,6 & 58,6 & $58.6(6)$ & 2,0 & $2.0(6)$ & C15-C16-C17-C18 & $-38,7$ & $-39,0$ & $-39.0(8)$ & 0,3 & $0.3(8)$ \\
\hline $\mathrm{C} 2-\mathrm{C} 1-\mathrm{C} 10-\mathrm{C} 5$ & $-56,0$ & $-56,4$ & $-56.4(6)$ & 0,4 & $0.4(6)$ & C16-C17-C18-C13 & 0,0 & 0,4 & $0.4(7)$ & 0,4 & $0.4(7)$ \\
\hline $\mathrm{C} 10-\mathrm{C} 1-\mathrm{C} 2-\mathrm{C} 3$ & 49,0 & 51,4 & $51.4(7)$ & 2,4 & $2.4(7)$ & C14-C13-C18-C17 & 51,1 & 52,4 & $52.4(6)$ & 1,3 & $1.3(6)$ \\
\hline Ring B & & & & & & Ring E & & & & & \\
\hline C6-C5-C10-C9 & $-49,0$ & $-51,8$ & $-51.9(6)$ & 2,9 & $3.0(6)$ & C17-C18-C19-C20 & $-46,5$ & $-47,2$ & $-47.1(7)$ & 0,7 & $0.6(7)$ \\
\hline C10-C5-C6-C7 & 48,0 & 51,1 & $51.1(6)$ & 3,1 & $3.1(6)$ & C22-C17-C18-C19 & $-7,5$ & $-7,1$ & $-7.1(7)$ & 0,4 & $0.4(7)$ \\
\hline C5-C6-C7-C8 & $-56,9$ & $-57,6$ & $-57.6(6)$ & 0,7 & $0.7(6)$ & $\mathrm{C} 18-\mathrm{C} 17-\mathrm{C} 22-\mathrm{C} 21$ & 57,9 & 58,7 & $58.7(7)$ & 0,8 & $0.8(7)$ \\
\hline C6-C7-C8-C9 & 63,2 & 59,6 & $59.7(6)$ & 3,6 & $3.7(6)$ & C20-C21-C22-C17 & $-55,4$ & $-57,0$ & $-57.1(7)$ & 1,6 & $1.7(7)$ \\
\hline $\mathrm{C} 7-\mathrm{C} 8-\mathrm{C} 9-\mathrm{C} 10$ & $-60,2$ & $-56,1$ & $-56.2(6)$ & 4,1 & $4.2(6)$ & C19-C20-C21-C22 & 1,3 & 2,2 & $2.2(8)$ & 0,8 & $0.8(8)$ \\
\hline C8-C9-C10-C5 & 54,9 & 55,0 & $55.0(6)$ & 0,1 & $0.1(6)$ & C18-C19-C20-C21 & 49,8 & 49,5 & $49.5(7)$ & 0,3 & $0.3(7)$ \\
\hline Ring C & & & & & & Ring & $r^{\mathrm{b}}$ & & & & \\
\hline C8-C9-C11-C12 & $-48,6$ & $-47,7$ & $-47.8(6)$ & 0,9 & $1.0(6)$ & A & 1,9 & & & & \\
\hline C14-C8-C9-C10 & 165,3 & 167,1 & $167.1(5)$ & 1,8 & $1.8(5)$ & $\mathrm{B}$ & 2,8 & & & & \\
\hline C9-C8-C14-C13 & $-54,5$ & $-51,4$ & $-51.5(6)$ & 3,1 & $3.2(6)$ & $\mathrm{C}$ & 1,6 & & & & \\
\hline C12-C13-C14-C8 & 55,2 & 54,1 & $54.1(6)$ & 1,1 & $1.1(6)$ & $\mathrm{D}$ & 2,0 & & & & \\
\hline C11-C12-C13-C14 & $-58,4$ & $-58,1$ & $-58.1(6)$ & 0,3 & $0.3(6)$ & $\mathrm{E}$ & 0,9 & & & & \\
\hline C9-C11-C12-C13 & 57,3 & 56,5 & $56.5(6)$ & 0,8 & $0.8(6)$ & & $1,5^{\mathrm{c}}$ & & & & \\
\hline
\end{tabular}

${ }^{(a)} \varepsilon_{1}=\mid$ calc-SDPD $\left|; \varepsilon_{2}=\right|$ calc-MC $\mid ;{ }^{(\mathrm{b})} r=\left[1 / n\left(\sum_{i=1}^{n} \varepsilon_{i}^{2}\right)\right]^{1 / 2} n=6 ;{ }^{(\mathrm{c})} 1 / n\left(\sum_{i=1}^{n} r_{i}\right) n=5$

* Parenthesized figures represent the estimated standard deviation (esd) in terms of least units cited for the value to the immediate left. 
Table 4. Atomic fractional coordinates $\left(\mathrm{x}_{\text {exp }}, \mathrm{y}_{\text {exp }}, \mathrm{z}_{\text {exp }}\right)$ and related isotropic displacement $\mathrm{U}_{\mathrm{iso}}\left(\AA^{2}\right)$. The atomic fractional coordinates $\left(\mathrm{x}_{\text {mono }}, \mathrm{y}_{\text {mono }}, \mathrm{z}_{\mathrm{mon}}\right)$ and $\mathrm{Uiso}_{\text {mono }}$ were extracted from Table 1 in ref. 23

\begin{tabular}{|c|c|c|c|c|c|c|c|c|}
\hline Atom & $X_{\text {exp }}$ & $Y_{\exp }$ & $Z_{\text {exp }}$ & Uiso* & $X_{\text {mono }}$ & $Y_{\text {mono }}$ & $Z_{\text {mono }}$ & $U_{\text {Uiso }_{\text {mono }}}$ \\
\hline $\mathrm{O}(1)$ & 0,0859 & 0,2123 & 0,5126 & 0,05000 & 0.0876 & 0.2133 & 0.5126 & 0.0415 \\
\hline $\mathrm{C}(1)$ & 0,2208 & 0,2771 & 0,4003 & 0,05000 & 0.2206 & 0.2781 & 0.3997 & 0.0327 \\
\hline $\mathrm{C}(2)$ & 0,0671 & 0,2970 & 0,4401 & 0,05000 & 0.0649 & 0.2969 & 0.4394 & 0.0383 \\
\hline$C(3)$ & 0,0466 & 0,2104 & 0,4702 & 0,05000 & 0.0485 & 0.2105 & 0.4709 & 0.0323 \\
\hline $\mathrm{C}(4)$ & $-0,0091$ & 0,1184 & 0,4457 & 0,05000 & -0.0107 & 0.1183 & 0.4453 & 0.0317 \\
\hline $\mathrm{C}(5)$ & 0,1634 & 0,0964 & 0,4077 & 0,05000 & 0.1601 & 0.0960 & 0.4074 & 0.0290 \\
\hline$C(6)$ & 0,0918 & 0,0074 & 0,3786 & 0,05000 & 0.0928 & 0.0076 & 0.3788 & 0.0332 \\
\hline $\mathrm{C}(7)$ & 0,2163 & $-0,0085$ & 0,3340 & 0,05000 & 0.2179 & -0.0078 & 0.3337 & 0.0338 \\
\hline $\mathrm{C}(8)$ & 0,2059 & 0,0801 & 0,3018 & 0,05000 & 0.2041 & 0.0808 & 0.3017 & 0.0270 \\
\hline$C(9)$ & 0,2956 & 0,1711 & 0,3266 & 0,05000 & 0.3001 & 0.1704 & 0.3270 & 0.0263 \\
\hline$C(10)$ & 0,1703 & 0,1840 & 0,3727 & 0,05000 & 0.1691 & 0.1847 & 0.3732 & 0.0253 \\
\hline $\mathrm{C}(11)$ & 0,2699 & 0,2577 & 0,2949 & 0,05000 & 0.2662 & 0.2580 & 0.2949 & 0.0315 \\
\hline$C(12)$ & 0,3415 & 0,2417 & 0,2443 & 0,05000 & 0.3411 & 0.2425 & 0.2443 & 0.0308 \\
\hline$C(13)$ & 0,2294 & 0,1559 & 0,2202 & 0,05000 & 0.2333 & 0.1570 & 0.2197 & 0.0260 \\
\hline$C(14)$ & 0,2713 & 0,0637 & 0,2496 & 0,05000 & 0.2714 & 0.0638 & 0.2496 & 0.0279 \\
\hline$C(15)$ & 0,1375 & $-0,0195$ & 0,2278 & 0,05000 & 0.1392 & -0.0194 & 0.2283 & 0.0368 \\
\hline$C(16)$ & 0,0992 & $-0,0124$ & 0,1746 & 0,05000 & 0.0977 & -0.0125 & 0.1746 & 0.0432 \\
\hline $\mathrm{C}(17)$ & 0,2528 & 0,0472 & 0,1441 & 0,05000 & 0.2517 & 0.0471 & 0.1442 & 0.0355 \\
\hline$C(18)$ & 0,3258 & 0,1421 & 0,1695 & 0,05000 & 0.3261 & 0.1430 & 0.1693 & 0.0288 \\
\hline$C(19)$ & 0,2881 & 0,2319 & 0,1392 & 0,05000 & 0.2890 & 0.2322 & 0.1382 & 0.0360 \\
\hline$C(20)$ & 0,3632 & 0,2236 & 0,0862 & 0,05000 & 0.3648 & 0.2248 & 0.0870 & 0.0395 \\
\hline $\mathrm{C}(21)$ & 0,2804 & 0,1314 & 0,0645 & 0,05000 & 0.2751 & 01319 & 0.0650 & 0.0527 \\
\hline$C(22)$ & 0,1390 & 0,0723 & 0,0987 & 0,05000 & 0.1387 & 0.0741 & 0.0980 & 0.0466 \\
\hline $\mathrm{C}(23)$ & 0,6093 & 0,2258 & 0,0862 & 0,05000 & -0.0548 & 0.0370 & 0.4800 & 0.0472 \\
\hline$C(24)$ & 0,2843 & 0,3121 & 0,0606 & 0,05000 & 0.3694 & 0.0752 & 0.4326 & 0.0384 \\
\hline$C(25)$ & 0,4410 & $-0,0177$ & 0,1313 & 0,05000 & 0.5384 & 0.1648 & 0.3376 & 0.0371 \\
\hline$C(26)$ & 0,0008 & 0,1822 & 0,2172 & 0,05000 & -0.0052 & 0.1819 & 0.2168 & 0.0339 \\
\hline$C(27)$ & 0,5049 & 0,0328 & 0,2480 & 0,05000 & 0.5050 & 0.0323 & 0.2484 & 0.0356 \\
\hline$C(28)$ & 0,5415 & 0,1628 & 0,3377 & 0,05000 & 0.4380 & -0.0176 & 0.1306 & 0.0470 \\
\hline C(29) & 0,3705 & 0,0751 & 0,4321 & 0,05000 & 0.2839 & 0.3126 & 0.0610 & 0.0583 \\
\hline$C(30)$ & $-0,0579$ & 0,0381 & 0,4813 & 0,05000 & 0.6067 & 0.2274 & 0.0858 & 0.0631 \\
\hline
\end{tabular}

${ }^{*}$ ) Due to the reduced number of independent reflections for an usual powder diffraction, 284 in this work, the hydrogen $\left(0.06000 \AA^{2}\right)$ carbon and oxygen $(0.05000$ $\AA^{2}$ ) displacements were based as reported elsewhere in literature.

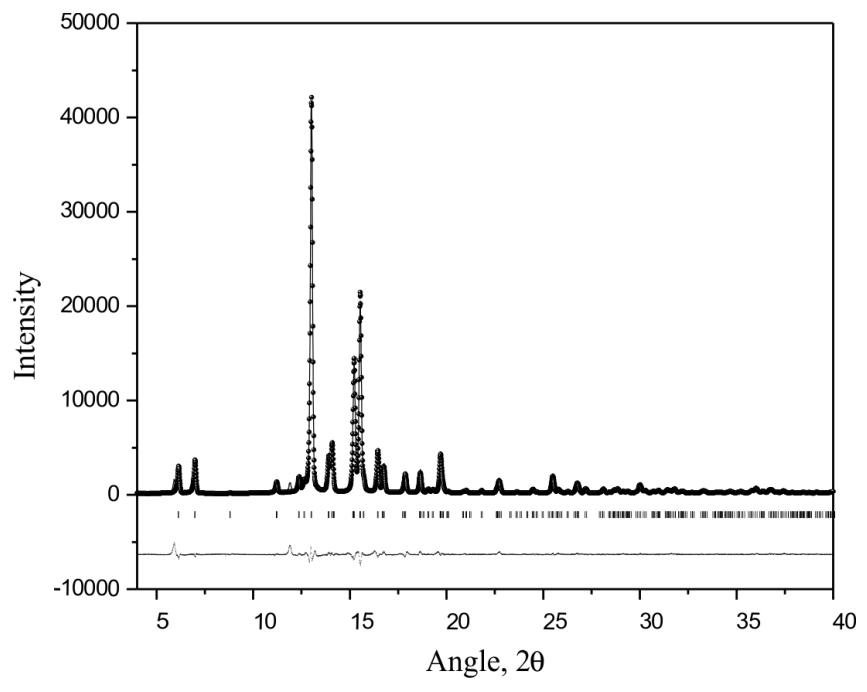

Figure 4. Final Rietveld refinement plot of friedelin. The observed data are shown by dots (.) and the calculated pattern represented by a solid line (-). The difference is plotted below; vertical bars indicate the Bragg reflection positions (।)

\section{CONCLUSION}

This study using SDPD revealed that the rings A, B and C of friedelin adopt a chair conformation and that the cis-decalin D/E system has a boat-boat conformation. The success of this SDPD was based on the purity grade (>98\%) of friedelin. The correlations between SDPD and DFT calculations were significantly higher, indicating that the experimental crystallographic solution details of the molecular conformation of friedelin were reproduced with excellent agreement by the gas phase DFT calculations. It is important to point it out that the degenerated conformation was based on no solute-solvent interaction since it is obtained in gas phase. These interactions would be responsible for the final crystal conformation.

Based on the results found in this work, it can be concluded that Crystalline Powder X-ray Diffraction represents an adequate alternative for application in conformational studies of PCTT and other organic compounds.

\section{ACKNOWLEDGMENTS}

The authors do thank the Fundação de Amparo à Pesquisa de Minas Gerais (FAPEMIG) and Fundação de Amparo à Pesquisa do Estado da Bahia (FAPESB) for financial and scholarship support. 


\section{REFERENCES}

1. Oliveira, D. M.; Silva, G. D. F.; Duarte, L. P.; Vieira Filho, S. A.; Guimarães, L.; Duarte, H. A.; De Souza, E.; Struct. Chem. 2009, 20, 1005.

2. Duarte, L. P.; Miranda, R. R. S.; Rodrigues, S. B. V.; Silva, G. D. F.; Vieira Filho, S. A.; Knupp, V. F.; Molecules 2009, 14, 598.

3. Tedesco, E.; Turner, G. W.; Harris, K. D. M.; Johnston, R. I.; Kariuki, B. M.; Angew. Chem., Int. Ed. 2000, 39, 4488.

4. David, W. I. F.; Shankland, K.; Mccusker, L. B.; Baerlocher, C. H.; Structure Determination from Powder Diffraction Data, Oxford University Press Inc.: New York, 2002.

5. David, W. I. F.; Shankland, K.; Acta Crystallogr., Sect. A: Found. Crystallogr. 2008, 64, 52.

6. Becke, A. D.; Phys. Rev. A: At., Mol., Opt. Phys. 1988, 38, 3098.

7. Becke, A. D.; J. Chem. Phys. 1993, 98, 5648.

8. Mo, F.; Winther, S.; Scrimgeour, S. N.; Acta Crystallogr., Sect. B: Struct. Sci. 1989, B45, 261.

9. Gottlieb, H. E.; Ramaiah, P. A.; Lavie, D.; Magn. Reson. Chem. 1985, 23,616 .

10. Mahato, S. B.; Kundu, A. P.; Phytochemistry 1994, 37, 1517.

11. Sen-Gupta, H. K.; Tucker, S. H.; J. Chem. Soc., Trans. 1922, 121, 557.

12. Chauham, S. M. S.; Singh, M.; Kumar, A.; Indian J. Chem., Sect. B: Org. Chem. Incl. Med. Chem. 2004, 43, 223.

13. Frisch, M. J.; Trucks, G. W.; Schlegel, H. B.; Scuseria, G. E.; Robb, M. A.; Cheeseman, J. R.; Montgomery Jr., J. A.; Vreven, T.; Kudin, K. N.; Burant, J. C.; Millam, J. M.; Iyengar, S. S.; Tomasi, J.; Barone, V.; Mennucci, B.; Cossi, M.; Scalmani, G.; Rega, N.; Petersson, G. A.; Nakatsuji, H.; Hada, M.; Ehara, M.; Toyota, K.; Fukuda, R.; Hasegawa, J.; Ishida, M.; Nakajima, T.; Honda, Y.; Kitao, O.; Nakai, H.; Klene, M.; Li, X.; Knox, J. E; Hratchian, H. P.; Cross, J. B.; Bakken, V.; Adamo, C.; Jaramillo, J.; Gomperts, R.; Stratmann, R. E.; Yazyev, O.; Austin, A.
J.; Cammi, R.; Pomelli, C.; Ochterski, J. W.; Ayala, P. Y.; Morokuma, K.; Voth, G. A.; Salvador, P.; Dannenberg, J. J.; Zakrzewski, V. G.; Dapprich, S.; Daniels, A. D.; Strain, M. C.; Farkas, O.; Malick, D. K.; Rabuck, A. D.; Raghavachari, K.; Foresman, J. B.; Ortiz, J. V.; Cui, Q.; Baboul, A. G.; Clifford, S.; Cioslowski, J.; Stefanov, B. B.; Liu, G.; Liashenko, A.; Piskorz, P.; Komaromi, I.; Martin, R. L.; Fox, D. J.; Keith, T.; Al-Laham, M. A.; Peng, C. Y.; Nanayakkara, A.; Challacombe, M.; Gill, P. M. W.; Johnson, B.; Chen, W.; Wong, M. W.; Gonzalez, C.; Pople, J. A.; Gaussian 03, Revision D.01; Gaussian, Inc., Wallingford CT, 2004.

14. Ditchfield, R.; Hehre, W. J.; Pople, J. A.; J. Chem. Phys. 1971, 54, 724.

15. Hariharan, P. C.; Pople, J. A.; Mol. Phys. 1974, 27, 209.

16. Mcquarrie, D. A.; Simon, J. D.; Molecular Thermodynamics, University Science Books: Sausalito, 1999.

17. Masaki, N.; Mineo, N.; Kikuchi, T.; J. Chem. Soc., Perkin Trans. 1975, 2,610 .

18. Jiang, R. W.; Ma, S. C.; He, Z. D.; Huang, X. S.; But, P. P. H.; Wang, H.; Chan, S. P.; Ooi, V. E. C.; Xu, H. X.; Mak, T. C. W.; Bioorg. Med. Chem. 2002, 10, 2161.

19. Cota, A. B.; Mascarenhas, Y. P.; Silva, G. D. F.; de Souza, J. R.; Acta Crystallogr., Sect. C: Cryst. Struct. Commun. 1990, 46, 326.

20. Boultif, A.; Louër, D.; J. Appl. Crystallogr. 1991, 24, 987.

21. Advanced Chemistry Development Inc.; ACD/Labs; Software for Analytical and Chemical Knowledge Management, Chemical Nomenclature, and In-Silico PhysChem and ADME-Tox; Canada, 1994.

22. Burnett, M. N.; Johnson, C. K.; ORTEP-III: Oak Ridge Thermal Ellipsoid Plot Program for Crystal Structure Illustrations, Oak Ridge National Laboratory Report ORNL-6895: Tennessee, 1996.

23. Declercq, B. J. P.; Puryvelde, L. V.; De Kimp, N.; Nage, M.; Verhegge, G.; De Vierman, R.; Acta Crystallogr., Sect. C: Cryst. Struct. Commun. 1991, 47, 209. 